\title{
Mutations in MT-ATP6 are a frequent cause of adult-onset spinocerebellar ataxia
}

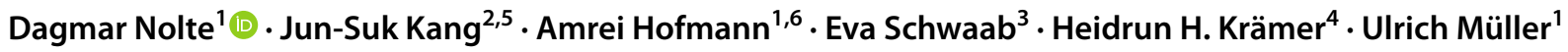

Received: 1 March 2021 / Revised: 6 May 2021 / Accepted: 8 May 2021 / Published online: 26 May 2021

(c) The Author(s) 2021

\begin{abstract}
Adult-onset ataxias are a genetically and clinically heterogeneous group of movement disorders. In addition to nuclear gene mutations, sequence changes have also been described in the mitochondrial genome. Here, we present findings of mutation analysis of the mitochondrial gene MT-ATP6. We analyzed 94 patients with adult-onset spinocerebellar ataxia (SCA), including 34 sporadic cases. In all patients, common sequence changes found in SCAs such as repeat expansions and point mutations had been excluded previously. We found pathogenic MT-ATP variants in five of these patients (5.32\%), two of whom were sporadic. Four of the five mutations have not previously been described in ataxias. All but one of these mutations affect transmembrane helices of subunit- $\alpha$ of ATP synthase. Two mutations (p.G16S, and p.P18S) disrupt transmembrane helix 1 (TMH1), one mutation (p.G167D) affects TMH5, and another one (p.L217P) TMH6. The fifth mutation (p.T96A) describes an amino acid change in close proximity to transmembrane helix 3 (TMH3). The level of heteroplasmy was either complete or very high ranging from 87 to $99 \%$. The high prevalence of pathogenic MT-ATP6 variants suggests that analysis of this gene should be included in the routine workup of both hereditary and sporadic ataxias.
\end{abstract}

Keywords Adult-onset ataxia $\cdot M T-A T P 6 \cdot$ ATP synthase $\cdot$ Complex V defect

\section{Introduction}

Hereditary adult-onset ataxias are a phenotypically and genetically heterogeneous group of movement disorders. They can be transmitted as autosomal-dominant, autosomalrecessive, X-linked, or mitochondrial traits. Autosomal-dominant spinocerebellar ataxias (SCA) are characterized by gait and limb ataxia, associated with dysarthria and abnormal eye movements in most patients. Additional signs and symptoms

Dagmar Nolte

dagmar.nolte@humangenetik.med.uni-giessen.de

1 Institut für Humangenetik, Justus-Liebig-Universität Giessen, Schlangenzahl 14, Giessen 35392, Germany

2 Klinikum der Johann Wolfgang Goethe-Universität, Klinik für Neurologie, Frankfurt, Germany

3 Praxis für Humangenetik, Wiesbaden, Germany

4 Klinik für Neurologie, Justus-Liebig-Universität Giessen, Giessen, Germany

5 Present Address: Neuropraxis, Frankfurt, Germany

6 Present Address: Klinikum Worms, Klinik für Pädiatrie, Worms, Germany may comprise aberrant reflexes, seizures, dystonia, tremor, myoclonus, and cognitive impairment. Mutations have been described in various genes in SCAs. The types of mutations observed are repeat expansions, point mutations, deletions, and insertions in nuclear genes [1]. No obvious genotype/ phenotype correlations can be established in most cases. Exceptions include SCA7 characterized by ataxia concurring with retinopathy, and SCA34 that frequently presents with erythrokeratodermia in addition to ataxia [1].

Mutations of mitochondrial DNA frequently underlie ataxia-associated syndromes, even if ataxia is not the major sign [2-4]. One of the genes affected, mitochondrial ATP synthase 6 (MT-ATP6), codes for ATP synthase subunit- $\alpha$ which is a subunit of the $\mathrm{F}_{1} \mathrm{~F}_{0} \mathrm{ATP}$-synthase complex responsible for mitochondrial energy production [5].

MT-ATP6 mutations including point mutations, deletions and truncations have also been described in adult-onset ataxia patients. The phenotype of ataxia caused by mutations in MT-ATP6 can frequently not be distinguished from ataxias caused by nuclear gene mutations [6]. In other cases, however, ataxia is associated with various symptoms such as combinations of ataxia with spastic paraplegia [7], motor neuron disease [8], neuropathy [9], myeloneuropathy [10], 
white matter abnormalities, kidney disease and cognitive decline [11], peripheral neuropathy, diabetes and hypergonadotropic hypogonadism [12], and episodic weakness combined with inherited axonal neuropathy [13]. Of these syndromes, only the complex ataxia-related syndrome described by Kytövouri is caused by a unique mutation of MT-ATP6, m. $8561 \mathrm{C}>\mathrm{G}$ (p.P12S) [12], which was formerly not associated with maternally inherited Leigh syndrome (MILS), or neuropathy, ataxia, and retinitis pigmentosa (NARP) syndrome [3].

The degree of heteroplasmy of the mutated gene $M T$ ATP6 facilitates classification of some mitochondrial syndromes. Thus, a mutation load of $>90 \%$ is frequently found in MILS syndrome [3, 14] and MT-ATP6 mutations in $70-90 \%$ of mitochondrial DNA often cause NARP syndrome $[2,3,15,16]$.

The following study was performed to determine the relative frequency and possible specificity of MT-ATP6 mutations in patients clinically classified as adult-onset spinocerebellar ataxia.

\section{Patients and methods}

\section{Genetic analysis}

Ninety-four unrelated spinocerebellar ataxia patients were tested for mutations in MT-ATP6 (ENSG00000198899). Eighty-six patients were of German origin, three were Russians, two Polish, and one patient each came from Turkey, Spain, and Italy. The study was approved by the Ethics Committee of the University of Giessen. Patients gave written informed consent according to the guidelines of the German Genetics Diagnostics Act. All patients were examined and diagnosed at specialized German movement disorder centers. Other causes of ataxic movement disorders such as neoplasia, stroke, CNS infection, multiple sclerosis, vitamin deficiency, and alcohol abuse were excluded in all patients. Sixty patients had a positive family history consistent with autosomal-dominant or mitochondrial inheritance. Thirtyfour patients were classified as sporadic.

DNA was extracted from peripheral blood. Repeat expansions at loci SCA1-3, SCA6-8, SCA10, SCA12, and SCA17 were excluded. Similarly, no pathogenic variants were detected at loci SCA11 (TTBK2), SCA13 (KCNC3), SCA14 (PRKCG), SCA19 (KCND3), SCA23 (PDYN), SCA27 (FGF14), SCA28 (AGF3L2), and SCA38 (ELOVL5). Large deletions at SCA15/16 (ITPRI/SUMF1) were excluded by quantitative PCR.

A 953-bp fragment of MT-ATP6 was amplified by PCR using primers mtATP6_F: 5'-GCCCACCATAATTACCCC
-3', and mtATP6_R: 5'-GCCTAGTATGAGGAGCGTTATG$3^{\prime}$. PCR fragments were sequenced in both directions.

\section{Analysis of degree of heteroplasmy}

Heteroplasmy levels for m.8572G $>$ A (p.G16S), m.8578C > T (p.P18S), m.8812A > G (p.T96A), m.9026G > A (p.G167D), and m.9176 T > C (p.L217P) were determined by pyrosequencing as described earlier [6]. DNA of the five patients was amplified by PCR to generate short products. One of the primers was biotinylated to facilitate isolation of the template strand via streptavidin. For pyrosequencing, a sequencing primer was used in close proximity to the mutation. Pyromark Assay Design Software v.2.0 (Qiagen/Hilden) was used for design of the variant-specific assays. Pyrosequencing was done on a Pyromark Q24 sequencer according to the manufacturer's instructions. Assays were repeated at least twice.

A single PCR product was generated for closely adjacent variants $\mathrm{m} .8572 \mathrm{G}>\mathrm{A}$ (p.G16S) and m.8578C $>\mathrm{T}$ (p.P18S). Primers were Pyro_G16S_P18S_F: 5'-TCT GTTCGCTTCATTCATTGC-3' and 5'-biotinylated reverse primer Pyro_G16S_P18S_R: 5'-GAGGGGGAA ATAGAATGATCAGTA-3'. Both variants were quantified in DNA of patient \#960 (m.8572G > A), or patient \#982 (m.8578C > T) using primer PyroSeq_G16_P18_F: 5'-TGCCCCCACAATCCT-3'.

Variant m.8812A $>$ G (p.T96A) was analyzed with 5'-biotinylated forward primer Pyro_T96A_F: 5'-CTC GGACTCCTGCCTCACT-3', and Pyro_T96A_R: 5'-CTG TGCCCGCTCATAAGG-3'. Reverse primer used for quantification was PyroSeq_T96A_R: 5'-GGCTAGGTTTAT AGATAGTT-3'.

Primers for variant m.9026G > A (p.G167D) were Pyro_G167D_F: 5'-AACCAATAGCCCTGGCCGTAC3' and 5'-biotinylated Pyro_G167D_R: 5'-CGCTTCCAA TTAGGTGCATGA-3'. Primer used for quantification was PyroSeq_G167D_F: 5'-CTAACCGCTAACATTACTG-3'.

Variant m.9176T $>C$ (p.L217P) was analyzed using primers Pyro_L217P_F: 5'- TCGCCTTAATCCAAGCCT AC-3', and 5'-biotinylated Pyro_L217P_R: 5'- ATTATG TGTTGTCGTGCAGGTAGA-3'. Quantification was performed with primer PyroSeq_L217P_F: 5'-CCTACGTTT TCACACTTC-3'.

\section{Prediction of pathogenicity}

Pathogenicity of observed variants was analyzed in silico (Table 1). Programs used were MutationTaster2 (http:// www.mutationtaster.org) [17], Polyphen-2 (http://genetics. bwh.harvard.edu/pph2/) [18], PROVEAN (http://provean. jcvi.org) [19], SIFT (https://sift.bii.a-star.edu.sg) [20, 21], 
Table 1 Prediction, MitoMap frequency, and ACMG classification of MT-ATP6 missense variants detected in a cohort of 94 SCA patients

\begin{tabular}{|c|c|c|c|c|c|c|c|c|c|}
\hline $\begin{array}{l}\text { mDNA/cDNA/ } \\
\text { protein change/ } \\
\text { cases in cohort }\end{array}$ & $\begin{array}{l}\text { SNV number } \\
\text { MAF (ALFA } \\
\text { database) }\end{array}$ & $\begin{array}{l}\text { MutationTaster } \\
\text { (Score) }\end{array}$ & $\begin{array}{l}\text { Polyphen-2 } \\
\text { (Score) }\end{array}$ & $\begin{array}{l}\text { PROVEAN } \\
\text { (Score) }\end{array}$ & SIFT (Score) & $\begin{array}{l}\text { VEP/ } \\
\text { Ensen } \\
\text { SIFT / } \\
\text { phen }\end{array}$ & $\begin{array}{l}\text { lbl } \\
\text { (Poly- } \\
\text { Score) }\end{array}$ & $\begin{array}{l}\text { MitoMap } \\
\text { frequency }\end{array}$ & $\begin{array}{l}\text { ACMG Classifi- } \\
\text { cation criteria }\end{array}$ \\
\hline$\frac{\frac{\mathrm{m} .8572 \mathrm{G}>\mathrm{A}}{\mathrm{c} .46 \mathrm{G}>\mathrm{A},}}{\underline{\text { p.G16S. }}}$ & $\begin{array}{l}\text { rs } 28502681 \\
A=0.0009\end{array}$ & $\begin{array}{l}\text { Disease causing } \\
(0.9961)\end{array}$ & $\begin{array}{l}\text { Probably dam- } \\
\text { aging } \\
(0.895)\end{array}$ & $\begin{array}{l}\text { Deleterious } \\
(-4.623)\end{array}$ & $\begin{array}{l}\text { not tolerated } \\
(0.02)\end{array}$ & 0.03 & 0.498 & $0.344 \%$ & $\begin{array}{c}\text { Class } 3 \text { (variant } \\
\text { of uncertain } \\
\text { significance) } \\
\text { PM1, PP3 }\end{array}$ \\
\hline $\begin{array}{l}\frac{\mathrm{m} .8578 \mathrm{C}>\mathrm{T}}{\mathrm{c} .52 \mathrm{C}>\mathrm{T},} \\
\text { p.P18S, } \\
\underline{1}\end{array}$ & $\begin{array}{l}\text { rs } 1556423492 \\
\mathrm{~T}=0.0004\end{array}$ & $\begin{array}{l}\text { Polymorphism } \\
(0.9517)\end{array}$ & $\begin{array}{l}\text { Probably dam- } \\
\text { aging } \\
(0,999)\end{array}$ & $\begin{array}{l}\text { Deleterious } \\
(-6.594)\end{array}$ & $\begin{array}{l}\text { Tolerated } \\
(0.17)\end{array}$ & 0.03 & 0.996 & $0.058 \%$ & $\begin{array}{l}\text { Class } 4 \text { (likely } \\
\text { pathogenic) } \\
\text { PS4, PM1, PP3 }\end{array}$ \\
\hline $\begin{array}{l}\text { m. } 8584 \mathrm{G}>\mathrm{A} \\
\text { c.58G }>\mathrm{A}, \\
\text { p.A20T, } \\
2\end{array}$ & $\begin{array}{l}\mathrm{rs} 3135028 \\
\mathrm{~A}=0.0067\end{array}$ & $\begin{array}{l}\text { Polymorphism } \\
(0.9999)\end{array}$ & $\begin{array}{l}\text { Benign } \\
(0.004)\end{array}$ & $\begin{array}{l}\text { Neutral } \\
(-0.404)\end{array}$ & $\begin{array}{l}\text { Tolerated } \\
(0.21)\end{array}$ & 0.24 & 0.012 & $5.558 \%$ & $\begin{array}{l}\text { Class } 1 \text { (benign) } \\
\text { BA1 }\end{array}$ \\
\hline $\begin{array}{l}\text { m. } 8701 \mathrm{~A}>\mathrm{G} \\
\text { c. } 175 \mathrm{~A}>\mathrm{G}, \\
\text { p.T59A, } \\
3\end{array}$ & $\begin{array}{l}\text { rs } 2000975 \\
G=0.06433\end{array}$ & $\begin{array}{l}\text { Polymorphism } \\
(0.9999)\end{array}$ & $\begin{array}{l}\text { Benign } \\
(0.002)\end{array}$ & $\begin{array}{l}\text { Neutral } \\
(-0.935)\end{array}$ & $\begin{array}{l}\text { Tolerated } \\
(0.66)\end{array}$ & 0.51 & 0.005 & $32.975 \%$ & $\begin{array}{l}\text { Class } 1 \text { (benign) } \\
\text { BA1 }\end{array}$ \\
\hline $\begin{array}{l}\text { m. } 8705 \mathrm{~T}>\mathrm{C} \\
\text { c. } 179 \mathrm{~T}>\mathrm{C}, \\
\text { p.M60T, } \\
2\end{array}$ & $\begin{array}{l}\text { rs } 878959404 \\
C=0.0043\end{array}$ & $\begin{array}{l}\text { Polymorphism } \\
(0,9999)\end{array}$ & $\begin{array}{l}\text { benign } \\
(0.000)\end{array}$ & $\begin{array}{l}\text { Neutral } \\
(0.320)\end{array}$ & $\begin{array}{l}\text { tolerated } \\
(0.30)\end{array}$ & 0.68 & 0.0 & $0.383 \%$ & $\begin{array}{l}\text { Class } 2 \text { (likely } \\
\text { benign) } \\
\text { BP4, BP6 }\end{array}$ \\
\hline $\begin{array}{l}\text { m. } 8723 \mathrm{G}>\mathrm{A} \\
\mathrm{c} .197 \mathrm{G}>\mathrm{A}, \\
\text { p.R66Q, } \\
1\end{array}$ & rs unknown & $\begin{array}{l}\text { Polymorphism } \\
(0.9997)\end{array}$ & $\begin{array}{l}\text { Benign } \\
(0.021)\end{array}$ & $\begin{array}{l}\text { Neutral } \\
(-0.523)\end{array}$ & $\begin{array}{l}\text { Tolerated } \\
(0.51)\end{array}$ & 0.55 & 0.012 & $0.159 \%$ & $\begin{array}{l}\text { Class } 2 \text { (likely } \\
\text { benign) } \\
\text { BP4, BP6 }\end{array}$ \\
\hline $\begin{array}{l}\text { m. } 8764 \mathrm{G}>\mathrm{A} \\
\text { c. } 238 \mathrm{G}>\mathrm{A}, \\
\text { p.A80T } \\
1\end{array}$ & $\begin{array}{l}\text { rs } 1556423534 \\
A=0.0018\end{array}$ & $\begin{array}{l}\text { Polymorphism } \\
(0.9999)\end{array}$ & $\begin{array}{l}\text { Benign } \\
(0.001)\end{array}$ & $\begin{array}{l}\text { Neutral } \\
(-1.221)\end{array}$ & $\begin{array}{l}\text { Tolerated } \\
(0.26)\end{array}$ & 0.12 & 0.007 & $0.207 \%$ & $\begin{array}{l}\text { Class } 2 \text { (likely } \\
\text { benign) } \\
\text { BP4, BP6 }\end{array}$ \\
\hline $\begin{array}{l}\frac{\mathrm{m} .8812 \mathrm{~A}>\mathrm{G}}{\mathrm{c} \cdot 286 \mathrm{~A}>\mathrm{G}} \\
\frac{\text { p.T96A, }}{\underline{1}}\end{array}$ & $\begin{array}{l}\text { rs } 1556423543 \\
\mathrm{G}=0.0018\end{array}$ & $\begin{array}{l}\text { Polymorphism } \\
(0.9266)\end{array}$ & $\begin{array}{l}\text { Probably dam- } \\
\text { aging } \\
(0.994)\end{array}$ & $\begin{array}{l}\text { Deleterious } \\
(-3.891)\end{array}$ & $\begin{array}{l}\text { Not tolerated } \\
(0.03)\end{array}$ & 0.05 & 0.988 & $0.118 \%$ & $\begin{array}{c}\text { Class } 3 \text { (variant } \\
\text { of uncertain } \\
\text { significance) } \\
\text { PM1, PP3 }\end{array}$ \\
\hline $\begin{array}{l}\text { m. } 8950 \mathrm{G}>\mathrm{A} \\
\mathrm{c} .424 \mathrm{G}>\mathrm{A}, \\
\text { p.V142I, } \\
1\end{array}$ & $\begin{array}{l}\text { rs } 1556423574 \\
A=0.0008\end{array}$ & $\begin{array}{l}\text { Polymorphism } \\
(0.9999)\end{array}$ & $\begin{array}{l}\text { Benign } \\
(0.0)\end{array}$ & $\begin{array}{l}\text { Neutral } \\
(0.118)\end{array}$ & $\begin{array}{l}\text { Tolerated } \\
(1.0)\end{array}$ & 1.0 & 0.0 & $0.151 \%$ & $\begin{array}{l}\text { Class } 2 \text { (likely } \\
\text { benign) } \\
\text { BP4, BP6 }\end{array}$ \\
\hline $\begin{array}{l}\frac{\mathrm{m} .9026 \mathrm{G}>\mathrm{A}}{\mathrm{c} .500 \mathrm{G}>\mathrm{A}} \\
\text { p.G167D. } \\
\underline{1}\end{array}$ & COSV62293160 & $\begin{array}{l}\text { Disease causing } \\
(0.9999)\end{array}$ & $\begin{array}{l}\text { Probably dam- } \\
\text { aging } \\
(1.0)\end{array}$ & $\begin{array}{l}\text { Deleterious } \\
(-6.275)\end{array}$ & $\begin{array}{l}\text { Not tolerated } \\
(0.00)\end{array}$ & 0.0 & 0.999 & $0.006 \%$ & $\begin{array}{l}\text { Class } 5 \text { (patho- } \\
\text { genic) } \\
\text { PS1, PM1, PM2, } \\
\text { PM5 }\end{array}$ \\
\hline $\begin{array}{l}\text { m.9055G }>A \\
\text { c. } 529 \mathrm{G}>\mathrm{A}, \\
\text { p.A177T, } \\
7\end{array}$ & $\begin{array}{l}\text { rs } 193303045 \\
A=0.1556\end{array}$ & $\begin{array}{l}\text { Polymorphism } \\
(0.9988)\end{array}$ & $\begin{array}{l}\text { Probably dam- } \\
\text { aging } \\
(0.845)\end{array}$ & $\begin{array}{l}\text { Deleterious } \\
(-2.606)\end{array}$ & $\begin{array}{c}\text { Tolerated } \\
(0.16)\end{array}$ & 0.1 & 0.399 & $4.244 \%$ & $\begin{array}{l}\text { Class } 1 \text { (benign) } \\
\text { BS1, BS4 }\end{array}$ \\
\hline $\begin{array}{l}\text { m.9067A>G } \\
\text { c. } 541 \mathrm{~A}>\mathrm{G}, \\
\text { p.M181V, } \\
2\end{array}$ & rs unknown & $\begin{array}{l}\text { Polymorphism } \\
(0.9999)\end{array}$ & $\begin{array}{l}\text { Benign } \\
(0.003)\end{array}$ & $\begin{array}{l}\text { Neutral } \\
(-0.967)\end{array}$ & $\begin{array}{l}\text { Not tolerated } \\
(0.01)\end{array}$ & 0.01 & 0.007 & $0.070 \%$ & $\begin{array}{l}\text { Class } 2 \text { (likely } \\
\text { benign) } \\
\text { BP4, BP6 }\end{array}$ \\
\hline $\begin{array}{l}\text { m. } .9070 \mathrm{~T}>\mathrm{G} \\
\text { c. } 544 \mathrm{~T}>\mathrm{G}, \\
\text { p.S182A, } \\
1\end{array}$ & $\begin{array}{l}\text { rs } 879190502 \\
G=0.0020\end{array}$ & $\begin{array}{l}\text { Polymorphism } \\
(0.9999)\end{array}$ & $\begin{array}{l}\text { Benign } \\
(0.225)\end{array}$ & $\begin{array}{l}\text { Neutral } \\
(-0.122)\end{array}$ & $\begin{array}{l}\text { Tolerated } \\
(0.57)\end{array}$ & 0.12 & 0.182 & $0.126 \%$ & $\begin{array}{l}\text { Class } 2 \text { (likely } \\
\text { benign) } \\
\text { BP4, BP6 }\end{array}$ \\
\hline $\begin{array}{l}\frac{\mathrm{m} .9176 \mathrm{~T} C}{\mathrm{c} .650 \mathrm{~T}>\mathrm{C} .} \\
\frac{\mathrm{p} . \mathrm{L} 217 \mathrm{P}}{1}\end{array}$ & $\begin{array}{l}\text { rs199476135 } \\
\mathrm{C}=\mathrm{MAF} \text { unknown }\end{array}$ & $\begin{array}{l}\text { Disease causing } \\
(0.9999)\end{array}$ & $\begin{array}{l}\text { Probably dam- } \\
\text { aging } \\
(0.999)\end{array}$ & $\begin{array}{l}\text { Deleterious } \\
(-6.258)\end{array}$ & $\begin{array}{l}\text { Not tolerated } \\
(0.00)\end{array}$ & 0.0 & 0.998 & $0.006 \%$ & $\begin{array}{l}\text { Class } 5 \text { (patho- } \\
\text { genic) } \\
\text { PS1, PS3, PS4 }\end{array}$ \\
\hline
\end{tabular}

SCA spinocerebellar ataxia, MAF minor allele frequency, underline: likely pathogenic variants; ALFA database: (https://www.ncbi.nlm.nih.gov/ snp/docs/gsr/alfa) [36]; ACMG classification, detailed information is given in [24] 
and Variant Effect Predictor (VEP, https://www.ensembl. org/info/docs/tools/vep/index.html) [22], which is a modified version of a combination of SIFT and Polyphen-2 prediction programs.

Frequency of each variant detected (Table 1) was analyzed by searching the database MitoMap (https://www. mitomap.org) [23]. Variants were classified according to the ACMG guidelines [24] (Table 1).

\section{Clinical findings}

\section{Patient 1}

Disease onset in male patient 1 (\#982, family 1, II-2, Fig. 1a, Table 2) was at age 53 when he presented with gait instability and frequent falls. At age 56, comprehensive neurological examination revealed mild and slowly progressive gait ataxia, postural instability, dysdiadochokinesia, moderate horizontal nystagmus and mild dysarthria. Fine motor movements were not impaired. While psychiatric symptoms were excluded, the patient complained of moderate lack of concentration and forgetfulness. His older brother who had perinatal asphyxia presented with generalized dystonia and mild ataxic gait. His younger brother died at age 50 of unknown causes. However, a psychiatric disorder and tremor had been excluded. The patient's sister was healthy at her last examination at age 48 .
A

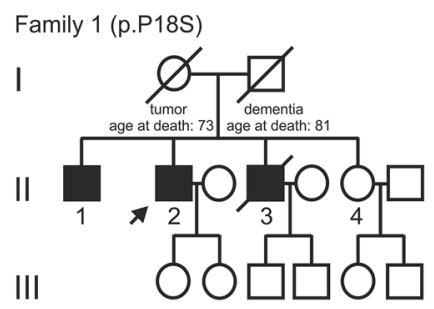

Family 2 (p.G167D)

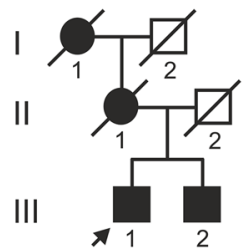

Index patient 3 (p.G16S)

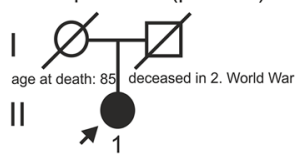

Index patient 4 (p.T96A)

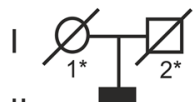

II

त 1

Index patient 5 (p.L217P)

I

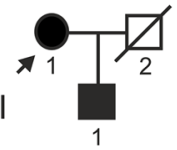

B

control
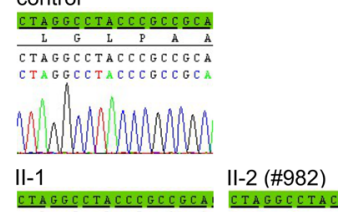

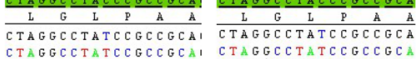

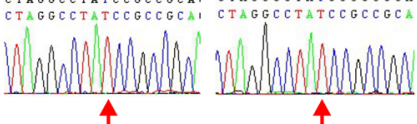

4
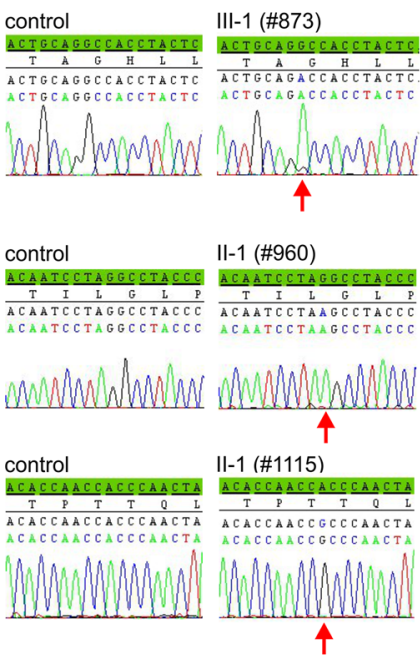

control

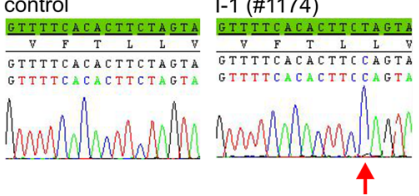

C

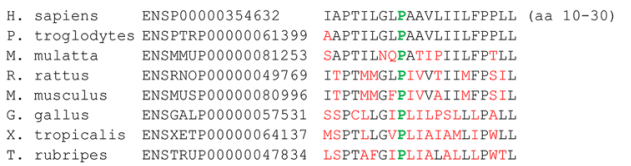

$\begin{array}{ll}x . & \text { tropicalis ENSXETP00000064137 } \\ \text { TSPTTLLGVPLIAIAMLIPWLI }\end{array}$

ENSP 00000354632 RLTANITAGHLLMHLIGSATL

M. mulatta ENSMMUP000000081253 RLTANITAGHLTMHLTGATI

R. rattus ENSRNOP00000049769 RLTANTTAGHLIMHLIGGATI

M. musculus ENSMUSP00000080996 RLTANITAGHLIMHLTGGATL

G. gallus ENSGALP00000057531 RLTANLTBGHLLIOLISTATI

. tropicalis ENSXETP00000064137 RLTANLTAGHLLIOLIATARE

r. rubripes ENSTRUP00000047834 RLTANLTAGHLLIQLIATAAE

H. sapiens ENSP00000354632 IAPTILGLPAAVLIILEPPLL (aа 10-30)

P. troglodytes ENSPTRP00000061399 AAPTIIGLPAAVLIILFPPLI

mulatta ENSMMUP00000081253 SAPTILNQPATIPIILFPTLL

rattus ENSRNOP00000049769 ITPTMMGLPIVVTIIMEPSIL

G. gallus ENSGAIP00000057531 SSPCLIGIPLILPSLLIPALI

X. tropicalis ENSXETP00000064137 MSPTLIGVPLIATAMLIPNLL

F. rubripes ENSTRUP00000047834 LSPTAFGIPLIALALLLPWTL

1. sapiens ENSP 00000354632 LPHSETPTTQLSMNLAMAIPL (aa 88-108)

P. troglodytes ENSPTRP00000061399 LPHSETPTTQLSMNLAMAIPI

M. mulatta ENSMMUP00000081253 MPYSETPTTQLSMNLAMAIPI

R. rattus ENSRNOP00000049769 LPHTETPTTQLSMNLSMAIPI

M. musculus ENSMUSP00000080996 LPHTETPTTQLSMNLSMAIPL

gallus ENSGALP00000057531 LPYTETPTTQLSMMMALALPL

tropicalis ENSXETP00000064137 LPYTETPTTQLSINMGLAVPI

rubripes ENSTRUP00000047834 LPYTETPTTQLSVNMALAVPL

H. sapiens ENSP00000354632 VALIQAYVFTLLVSLYLHDNT (aa 206-226)

P. troglodytes ENSPTRP00000061399 VALIQAYVFTLLVSLYLHDNT

M. mulatta ENSMMUP00000081253 VALIQAYVFTLIISLYLRNNT

R. rattus ENSRNOP00000049769 VALIQAYVFTLIVSLYLHDNT

M. musculus ENSMUSP00000080996 VALIQAYVFTLIVSLYLHDNT

G. gallus ENSGALP00000057531 VAMIQAYVFVLIISLYLEENI

tropicalis ENSXETP00000064137 VAMIQAYVFVLIISLYLQENV

rubripes ENSTRUP00000047834 VEMIQAYVFVLIISLYLQENV

Fig. 1 a Pedigrees of the German adult-onset SCA cases. Black symbols indicate affected probands. Index patients are marked by arrow. Probands for whom no clinical information was available are highlighted by an asterisk. b Electropherograms of sequences of index patients and controls. The relevant base changes are indicated by arrow. $\mathbf{c}$ Amino acid sequence alignments of ATP synthase subunit- $\alpha$ orthologs. Name of species and protein identifier numbers are given on the left. Amino acids mutated in patients are evolutionarily highly conserved and are highlighted in green. Non-conserved amino acid residues are given in red. H., Homo; P., Pan; M., Macaca; R., Rattus; M., Mus; G., Gallus; X., Xenopus; T., Takifugu 


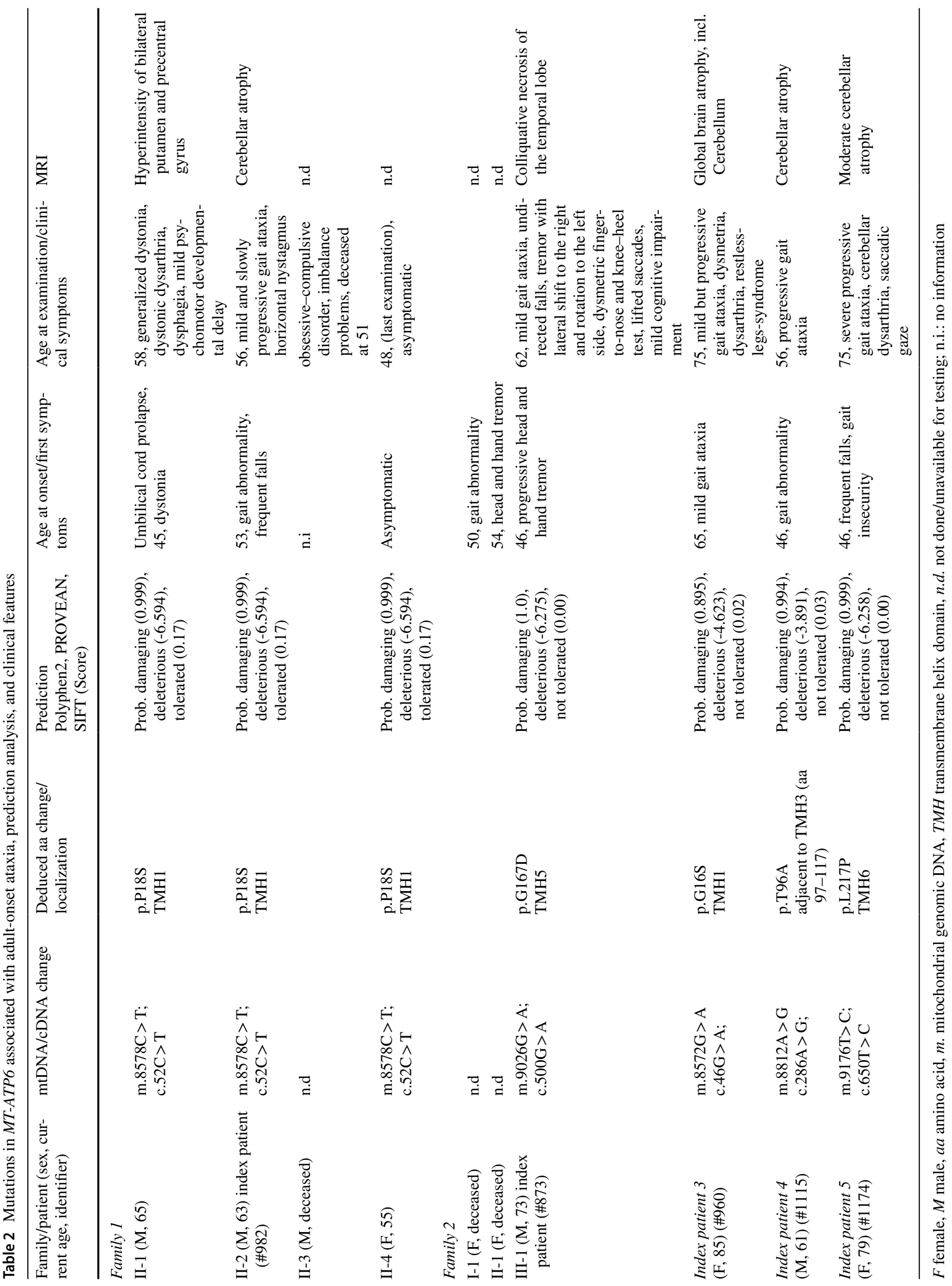




\section{Patient 2}

Male patient 2 (\#873, family 2, III-1, Fig. 1a, Table 2) came to clinical attention at age 62 because of ataxic gait, frequent falls, and tremor. Dysmetria was diagnosed by finger-to-nose and knee-heel test. The patient reported first occurrence of postural and action tremor of the hands at age 46. At the time of investigation, lifted saccades and abnormal executive function were diagnosed. Brain MRI revealed a colliquative necrosis of the temporal lobe. At age 73, ataxic wide-based gait had worsened. Tremor that was initially confined to the hands, now also affected the head and had become the major sign. SCA-loci that are associated with tremor (SCA12, and SCA15/16) have been excluded in this patient. The patient's younger brother, his deceased mother, and maternal grandmother had had similar signs and symptoms, of which tremor and mild ataxic gait were most striking.

\section{Patient 3}

Female patient 3 (\#960, II-1, Fig. 1a, Table 2) was sporadic with none of her parents affected. In the patient, a mild spinocerebellar ataxia was diagnosed at age 65 . The ataxia was progressive but did not affect the ability to walk without a cane for at least short distances at age 75 .

\section{Patient 4}

At age 56, sporadic male patient 4 (\#1115, II-1, Fig. 1a, Table 2) came to clinical attention due to a pure, progressive ataxic syndrome. MRI revealed distinct cerebellar atrophy. No health problems, in particular no movement disorders have been reported in his parents. His mother died at age 85 . His father was killed in World War II.

\section{Patient 5}

Abnormal gait and frequent falls first occurred in female patient 5 (\#1174, I-1, Fig. 1a, Table 2) at age 46. At age 75, a comprehensive neurological examination revealed pronounced dysarthria and a saccadic gaze sequence. Performance of directed movements and abnormal gait had severely worsened. Walking distance was only a few meters even when using a walker. MRI revealed distinct cerebellar atrophy. Her son suffered from similar symptoms that were diagnosed in his thirties.

\section{Results and discussion}

In 94 adult-onset SCA cases, we detected 14 variants of MT-ATP6 that result in non-synonymous amino acid (aa) changes (Table 1). Five of these variants were predicted to be deleterious by at least three of the five in silico tools applied (Table 1). These variants are $\mathrm{m} .8572 \mathrm{G}>\mathrm{A}$ (c.46G > A; p.G16S) detected in sporadic patient 3 (II-1, Table 2, Fig. 1b), m.8578C > T (c.52C > T; p.P18S) (patients II- 1 and II-2 of family 1, Table 2, Fig. 1b), m.8812A $>$ G (c.286A > G; p.T96A) (sporadic patient 4, II-1, Table 2, Fig. 1b), m.9026G $>$ A (c.500G $>$ A; p.G167D) (family 2 , patient III-1, Table 2, Fig. 1 b), and m.9176T $>$ C (c.650T > C; p.L217P) (patient 5, I-1, Table 2, Fig. 1b). An additional variant, m.9055G $>$ A (c.529G $>$ A; p.A177T), was classified as deleterious by two programs, but could be excluded, because it occurred multiple times in our collective and is also frequent in controls as reflected by the high MitoMap frequency of $4.24 \%$ (Table 1).

All deleterious variants but variant m.9176T $>C$ (c.650T > C; p.L217P) have not been associated with mitochondrial disease before. These variants were classified as class 5/pathogenic (m.9026G $>$ A, m.9176T $>$ C), class 4/ likely pathogenic $(\mathrm{m} .8578 \mathrm{C}>\mathrm{T})$, and class $3 /$ variant of uncertain significance $(\mathrm{m} .8572 \mathrm{G}>\mathrm{A}, \mathrm{m} .8812 \mathrm{~A}>\mathrm{G})$ according to the ACMG guidelines [24]. Pathogenicity of these variants is further supported by phylogenetic conservation of the affected aa residues (Fig. 1c), a finding that indicates an important role of these aa's in normal protein function.

Of the aa changes observed, all but one affect the helix structure of transmembrane domains of subunit- $\alpha$ of ATP synthase.

The two most proximal variants were detected in sporadic patient 3 (II-1), and in patients II-1, and II- 2 of family 1. Of these, $\mathrm{m} .8572 \mathrm{G}>\mathrm{A}(\mathrm{c} .46 \mathrm{G}>\mathrm{A})$ results in a glycine to serine change at aa position 16 (p.G16S). The mutation $\mathrm{m} .8578 \mathrm{C}>\mathrm{T}(\mathrm{c} .52 \mathrm{C}>\mathrm{T})$ of family 1 is located adjacent to $\mathrm{m} .8572$ and results in the substitution of a proline by a serine at aa position 18 (p.P18S). The pyrograms revealed homoplasmy for both $\mathrm{m} .8572 \mathrm{G}>\mathrm{A}$ (p.G16S), and $\mathrm{m} .8578 \mathrm{C}>\mathrm{T}$ (p.P18S) (Suppl. Figure 1). Both mutations affect the first transmembrane helix (TMH1) of subunit- $\alpha$ of ATP synthase and appear to disturb proton translocation. However, most disease-causing alterations of ATP synthase subunit- $\alpha$ appear to be located in the three distal transmembrane helices (TMH4-6) independent of the patient's phenotype [4, $13,25,26]$.

The mutation $\mathrm{m} .8561 \mathrm{C}>\mathrm{G}$ (p.P12R) of subunit- $\alpha$ in a patient with adult-onset ataxia, neuropathy, diabetes, and hypergonadotropic hypogonadism was shown to interfere with assembly of complex $\mathrm{V}$ of the mitochondrial respiratory chain by the alteration of two ATP synthase subunits. This results in impaired ATP synthesis [12].

In sporadic patient 4 (II-1), two non-synonymous aa changes were detected. Variant m.8723G $>$ A (c.197G $>$ A; p.R66Q) was predicted to be likely benign (Table 1). In contrast, variant m.8812A $>\mathrm{G}(\mathrm{c} .286 \mathrm{~A}>\mathrm{G})$ shows a mutation load of $97 \%$ (Suppl. Figure 1) and results in replacement of 
threonine by alanine at aa position 96 (p.T96A). This variant is located adjacent to TMH3, which spans aa 97-117 as shown in UniProtKB (http://www.uniprot.org/uniprot/ P00846).

Two variants were found in patient III- 1 of family 2 . Of these sequence changes, m.8950G $>$ A (c.424G $>$ A; p.V142I) was classified as likely benign (Table 1). In contrast, m.9026G $>$ A (c.500G $>$ A) is predicted to be pathogenic (Table 1). This mutation has a heteroplasmic load of about $87 \%$ (Suppl. Figure 1) and results in the replacement of a glycine by an aspartate at aa position 167 (p.G167D) of TMH5. A previous finding of an aa change at the same position (p.G167S) in patients with NARP-MILS syndrome [27] supports a possible impairment of the ATP synthase subunit $\alpha$. Recently, m.9026G $>$ A was also described in a child with intellectual disability, headaches, myalgias, and fatigue. However, a low mutation load of $16-23 \%$ in various tissues makes a correlation with the child's symptoms difficult [26].

Other deleterious aa changes associated with reduced ATP synthase activity have been described in close proximity to p.G167D. Among these, p.L170P was described in patients with cognitive delay, and early-onset ataxia [28]. p.L170P was also the first MT-ATP6 mutation associated with pure adult-onset ataxia [6]. Both our patient III-1 of family 2 carrying the p.G167D mutation and the patient described by Pfeffer [6] did not have cerebellar atrophy. In contrast to Pfeffer's and Sikorska's cases, the patient described here displayed a severe dystonic tremor. This finding shows that-similar to autosomal-dominant ataxia cases [1] - a strict genotype-phenotype correlation can also not be established in mitochondrial ataxia [3, 26, 29, 30].

Variant m.9176T $>$ C (c.650T $>$ C) was almost homoplasmic with a 99\% mutation load (Suppl. Figure 1) in patient 5 (I-1). The deduced amino acid change of leucine to proline at position 217 (p.L217P) is located in TMH6. Unlike the novel mutations described above, m.9176T $>\mathrm{C}$ has been reported at least 30 times in several disorders with highly variable disease duration and age of onset [4, 23, 30,31]. These disorders include a late-onset hereditary spastic paraplegia-like syndrome [7], MILS [32, 33], and ataxia in combination with familial bilateral striatal necrosis [34].

The five pathogenic variants of MT-ATP6 described here result in a prevalence of $5.32 \%$ in our group of adult-onset SCA patients. Two of these mutations occurred in the 34 patients with negative family histories, this amounts to $5.88 \%$ that is even higher than the overall prevalence in the cohort. The overall prevalence of $5.32 \%$ is significantly higher than the $3.13 \%$, that were reported by Pfeffer et al. in a study of 64 ataxia cases [6]. Our findings are in agreement with Pulkes' conjecture [35] of an important role and comparatively frequent occurrence of MT-ATP6 mutations in adult-onset ataxia patients.
In conclusion, MT-ATP6 mutations mainly affect the transmembrane helical domains of subunit- $\alpha$ of ATP synthase. Given the relatively frequent finding of MT-ATP 6 mutations in SCA patients, this gene should be routinely analyzed in SCA patients, even in the absence of positive family history, once repeat expansions have been excluded.

Supplementary Information The online version contains supplementary material available at https://doi.org/10.1007/s00415-021-10607-5.

Acknowledgements The authors thank all participating patients. We are indebted to Michaela Weiss and Sylvia Stanek for technical assistance.

Funding Open Access funding enabled and organized by Projekt DEAL. Nothing to report.

\section{Declarations}

Conflict of interest The authors declare no conflict of interest.

Ethical approval The study was performed according to the Declaration of Helsinki and approved by the ethical committee of the JustusLiebig-University.

Informed consent Informed consent was obtained from all participants in this study. Patients signed informed consent to publish their data. DNA samples are available from the corresponding author.

Open Access This article is licensed under a Creative Commons Attribution 4.0 International License, which permits use, sharing, adaptation, distribution and reproduction in any medium or format, as long as you give appropriate credit to the original author(s) and the source, provide a link to the Creative Commons licence, and indicate if changes were made. The images or other third party material in this article are included in the article's Creative Commons licence, unless indicated otherwise in a credit line to the material. If material is not included in the article's Creative Commons licence and your intended use is not permitted by statutory regulation or exceeds the permitted use, you will need to obtain permission directly from the copyright holder. To view a copy of this licence, visit http://creativecommons.org/licenses/by/4.0/.

\section{References}

1. Bird TD (1998) Hereditary Ataxia Overview. In: Pagon RA, Adam MP, Ardinger HH, et al., editors. GeneReviews®. Seattle (WA): University of Washington, Seattle; 1993-2020. http://www.ncbi. nlm.nih.gov/books/NBK1138/. Accessed 25 July 2019

2. Schon EA, DiMauro S, Hirano M (2012) Human mitochondrial DNA: roles of inherited and somatic mutations. Nat Rev Genet 13:878-890. https://doi.org/10.1038/nrg3275

3. Thorburn DR, Rahman S (2003) Mitochondrial DNA-Associated Leigh Syndrome and NARP. In: Pagon RA, Adam MP, Ardinger $\mathrm{HH}$, et al. editors. GeneReviews ${ }^{\circledR}$. Seattle (WA): University of Washington, Seattle; 1993-2020. http://www.ncbi.nlm.nih.gov/ books/NBK1173/. Accessed 28 Sept 2017.

4. Dautant A, Meier T, Hahn A, Tribouillard-Tanvier D, di Rago JP, Kucharczyk R (2018) ATP synthase diseases of mitochondrial genetic origin. Front Physiol 9:329. https://doi.org/10.3389/fphys. 2018.00329 
5. Anderson S, Bankier AT, Barrell BG, de Bruijn MH, Coulson AR, Drouin J, Eperon IC et al (1981) Sequence and organization of the human mitochondrial genome. Nature 290:457-465. https://doi. org/10.1038/290457a0

6. Pfeffer G, Blakely EL, Alston CL, Hassani A, Boggild M, Horvath R, Samuels DC et al (2012) Adult-onset spinocerebellar ataxia syndromes due to MTATP6 mutations. J Neurol Neurosurg Psychiatry 83:883-886. https://doi.org/10.1136/jnnp-2012-302568

7. Verny C, Guegen N, Desquiret V, Chevrollier A, Prundean A, Dubas F, Cassereau J et al (2011) Hereditary spastic paraplegialike disorder due to a mitochondrial ATP6 gene point mutation. Mitochondrion 11:70-75. https://doi.org/10.1016/j.mito.2010.07. 006

8. Brum M, Semedo C, Guerreiro R, Marques JP (2014) Motor neuron syndrome as a new phenotypic manifestation of mutation 9185T>C in Gene MTATP6. Case Rep Neurol Med. https://doi. org/10.1155/2014/701761

9. Rantamäki MT, Soini HK, Finnilä SM, Majamaa K, Udd B (2005) Adult-onset ataxia and polyneuropathy caused by mitochondrial 8993T->C mutation. Ann Neurol 58:337-340. https://doi.org/10. 1002/ana.20555

10. Bardakjian T, Scherer SS (2019) A MT-ATP6 mutation causes a slowly progressive myeloneuropathy. J Neuromuscul Dis 6:385387. https://doi.org/10.3233/jnd-190400

11. Bugiardini E, Bottani E, Marchet S, Poole OV, Beninca C, Horga A, Woodward C et al (2020) Expanding the molecular and phenotypic spectrum of truncating MT-ATP6 mutations. Neurol Genet 6:e381. https://doi.org/10.1212/nxg.0000000000000381

12. Kytövuori L, Lipponen J, Rusanen H, Komulainen T, Martikainen MH, Majamaa K (2016) A novel mutation m.8561C $>\mathrm{G}$ in MT-ATP6/8 causing a mitochondrial syndrome with ataxia, peripheral neuropathy, diabetes mellitus, and hypergonadotropic hypogonadism. J Neurol 263:2188-2195. https://doi.org/10.1007/ s00415-016-8249-2

13. Panosyan FB, Tawil R, Herrmann DN (2017) Episodic weakness and Charcot-marie-tooth disease due to a mitochondrial MT-ATP6 mutation. Muscle Nerve 55:922-927. https://doi.org/10.1002/mus. 25453

14. DiMauro S, Schon EA, Carelli V, Hirano M (2013) The clinical maze of mitochondrial neurology. Nat Rev Neurol 9:429-444. https://doi.org/10.1038/nrneurol.2013.126

15. Holt IJ, Harding AE, Petty RK, Morgan-Hughes JA (1990) A new mitochondrial disease associated with mitochondrial DNA heteroplasmy. Am J Hum Genet 46:428-433

16. Stendel C, Neuhofer C, Floride E, Yuqing S, Ganetzky RD, Park J, Freisinger P et al (2020) ATP6 Study Group. Delineating MTATP6-associated disease: from isolated neuropathy to early onset neurodegeneration. Neurol Genet 6:e393. https://doi.org/10.1212/ nxg.0000000000000393

17. Schwarz JM, Cooper DN, Schuelke M, Seelow D (2014) MutationTaster2: mutation prediction for the deep-sequencing age. Nat Methods 11:361-362. https://doi.org/10.1038/nmeth.2890

18. Adzhubei IA, Schmidt S, Peshkin L, Ramensky VE, Gerasimova A, Bork P, Kondrashov AS et al (2010) A method and server for predicting damaging missense mutations. Nat Methods 7:248249. https://doi.org/10.1038/nmeth0410-248

19. Choi Y, Chan AP (2015) PROVEAN web server: a tool to predict the functional effect of amino acid substitutions and indels. Bioinformatics 31:2745-2747. https://doi.org/10.1093/bioinformatics/ btv195

20. Kumar P, Henikoff S, Ng PC (2009) Predicting the effects of coding nonsynonymous variants on protein function using the SIFT algorithm. Nat Protoc 4:1073-1081. https://doi.org/10.1038/nprot. 2009.86

21. Sim NL, Kumar P, Jing Hu, Henikoff S, Schneider G, Ng PC (2012) SIFT web server: predicting effects of amino acid substitutions on proteins. Nucleic Acids Res 40:W452-W457. https://doi.org/10.1093/nar/gks539

22. McLaren W, Gil L, Hunt SE, Riat HS, Ritchie GR, Thormann A, Flicek P et al (2016) The Ensembl variant effect predictor. Genome Biol 17:122. https://doi.org/10.1186/s13059-016-0974-4

23. Lott MT, Leipzig JN, Derbeneva O, Xie HM, Chalkia D, Sarmady $\mathrm{M}$, Procaccio V et al (2013) mtDNA variation and analysis using MITOMAP and MITOMASTER. Curr Prot Bioinform. 44(1):23. https://doi.org/10.1002/0471250953.bi0123s44

24. Richards S, Aziz N, Bale S, Bick D, Das S, Gastier-Foster J, Grody WW et al (2015) ACMG Laboratory Quality Assurance Committee. Standards and guidelines for the interpretation of sequence variants: a joint consensus recommendation of the American College of Medical Genetics and Genomics and the Association for Molecular Pathology. Genet Med 17:405-424. https://doi.org/10. 1038/gim.2015.30

25. Xu T, Pagadala V, Mueller DM (2015) Understanding structure, function, and mutations in the mitochondrial ATP synthase. Microb Cell. 2: 105-125. https://doi.org/10.15698/mic2015.04. 197

26. Ganetzky RD, Stendel C, McCormick EM, Zolkipli-Cunningham Z, Goldstein AC, Klopstock T, Falk MJ (2019) MT-ATP6 mitochondrial disease variants: Phenotypic and biochemical features analysis in 218 published cases and cohort of 14 new cases. Hum Mutat 40:499-515. https://doi.org/10.1002/humu.23723

27. López-Gallardo E, Emperador S, Solano A, Llobet L, MartínNavarro A, López-Pérez MJ, Briones P et al (2014) Expanding the clinical phenotypes of MT-ATP6 mutations. Hum Mol Genet 1:6191-6200. https://doi.org/10.1093/hmg/ddu339

28. Sikorska M, Sandhu JK, Simon DK, Pathiraja V, Sodja C, Li Y, Ribecco-Lutkiewicz M et al (2009) Identification of ataxiaassociated mtDNA mutations ( $\mathrm{m} .4052 \mathrm{~T}>\mathrm{C}$ and $\mathrm{m} .9035 \mathrm{~T}>\mathrm{C}$ ) and evaluation of their pathogenicity in transmitochondrial cybrids. Muscle Nerve 40:381-394. https://doi.org/10.1002/mus.21355

29. Russell O, Turnbull D (2014) Mitochondrial DNA diseasemolecular insights and potential routes to a cure. Exp Cell Res 325:38-43. https://doi.org/10.1016/j.yexcr.2014.03.012

30. Rucheton B, Jardel C, Filaut S, Del Mar AA, Maisonobe T, Serre I, Romero NB et al (2020) Homoplasmic deleterious MT-ATP6/8 mutations in adult patients. Mitochondrion 55:64-77. https://doi. org/10.1016/j.mito.2020.08.004

31. Ng YS, Martikainen MH, Gorman GS, Blain A, Bugiardini E, Bunting A, Schaefer AM et al (2019) Pathogenic variants in MTATP6: A United Kingdom-based mitochondrial disease cohort study. Ann Neurol 86:310-315. https://doi.org/10.1002/ana.25525

32. Campos Y, Martín MA, Rubio JC, Solana LG, García-Benayas C, Terradas JL, Arenas J (1997) Leigh syndrome associated with the T9176C mutation in the ATPase 6 gene of mitochondrial DNA. Neurology 49:595-597. https://doi.org/10.1212/wnl.49.2.595

33. Dionisi-Vici C, Seneca S, Zeviani M, Fariello G, Rimoldi M, Bertini E, De Meirleir L (1998) Fulminant Leigh syndrome and sudden unexpected death in a family with the T9176C mutation of the mitochondrial ATPase 6 gene. J Inherit Metab Dis 21:2-8. https://doi.org/10.1023/a:1005397227996

34. Thyagarajan D, Shanske S, Vazquez-Memije M, De Vivo D, DiMauro S (1995) A novel mitochondrial ATPase 6 point mutation in familial bilateral striatal necrosis. Ann Neurol 38:468-472. https://doi.org/10.1002/ana.410380321

35. Pulkes T (2012) Adult-onset spinocerebellar ataxia due to MTATP6 mutations: are they more common than previously thought? J Neurol Neurosurg Psychiatry 83:857-858. https://doi. org/10.1136/jnnp-2012-302959

36. Phan L, Jin Y, Zhang H, Qiang W, Shekhtman E, Shao D, Revoe D, et al. (2020) "ALFA: Allele Frequency Aggregator." National Center for Biotechnology Information, U.S. National Library of Medicine http://www.ncbi.nlm.nih.gov/snp/docs/gsr/alfa/ 